\title{
Is dialysis hypotension caused by an abnormality of venous tone?
}

\author{
JOHN R BRADLEY, DAVID B EVANS, SHEILA M GORE, ALAN J COWLEY
}

\begin{abstract}
The role of peripheral vascular tone in the development of hypotension induced by dialysis was investigated in eight patients undergoing haemodialysis with acetate or bicarbonate buffered fluid. Each patient had two sessions of dialysis with acetate fluid and two with bicarbonate fluid in the order acetate, bicarbonate, bicarbonate, acetate or bicarbonate, acetate, acetate, bicarbonate. Mean arterial blood pressure fell at a mean rate of 3.9 $\mathrm{mm} \mathrm{Hg}$ /hour during dialysis with acetate fluid and $1.4 \mathrm{~mm} \mathrm{Hg} /$ hour during dialysis with bicarbonate fluid. The rate of fall was significantly greater during dialysis with acetate fluid compared with bicarbonate fluid. Heart rate increased by a mean rate of 2.6 beats/min/hour during dialysis with both acetate and bicarbonate fluid. Vascular resistance in the forearm increased at a rate of 3.6 units/hour during dialysis with acetate fluid and 4.5 units/hour during dialysis with bicarbonate fluid, but the venous bed of the forearm dilated. The index of venous tone rose at a mean rate of $0.23 \mathrm{ml} / 100 \mathrm{dl}$ over $40 \mathrm{~mm} \mathrm{Hg} /$ hour during dialysis with acetate fluid and $0.20 \mathrm{ml} / \mathrm{dl}$ over $40 \mathrm{~mm} \mathrm{Hg} /$ hour during dialysis with bicarbonate fluid.
\end{abstract}

Inappropriate peripheral venodilatation may be important in the development of hypotension induced by dialysis.

\section{Introduction}

Haemodialysis has revolutionised the treatment of patients with end stage renal failure; the morbidity associated with the procedure, however, is poorly recognised. Severe and symptomatic hypotension may occur in more than $25 \%$ of dialysis sessions and may restrict the amount of fluid that can be removed during dialysis. The cause of the hypotension is unknown but may be an autonomic neuropathy, whereby the patient's resistance vessels do not constrict appropriately to maintain blood pressure, or an inability to increase cardiac output by increasing heart rate. 'Another possible mechanism is excessive vasodilatation caused by acetate in the dialysis fluid. ${ }^{2}$ The clinical observation that administering small volumes of fluid may correct the hypotension ${ }^{3}$ does not support any of these proposed mechanisms and suggests that central plasma volume may be reduced excessively. In this study we examined changes in peripheral venous tone, which in part controls central plasma volume, and peripheral vascular resistance during dialysis and compared the changes that occur during dialysis with acetate and with bicarbonate fluids.

\section{Patients and methods}

Eight patients gave their informed consent to take part in the study, which was performed with the approval of the local ethical committee. The five

\footnotetext{
Department of Renal Medicine, Addenbrooke's Hospital, Cambridge CB2 2QQ

JOHN R BRADLEY, BMEDSCI, MRCP, clinical research fellow

DAVID B EVANS, FRCP, consultant physician
}

Biostatistics Unit, Medical Research Council Centre, Cambridge CB2 2BW SHEILA M GORE, MA, PHD, medical statistician

Department of Medicine, University Hospital, Nottingham NG7 2UH ALAN J COWLEY, MB, MRCP, lecturer in medicine

Correspondence to: Dr Bradley. men and three women had a mean age of $52($ SD 15.6) and had been receiving haemodialysis for $10.5(7 \cdot 6)$ months on average. None of the patients had evidence of cardiovascular disease and none was receiving cardiovascular drugs. The patients were studied during four dialysis sessions at weekly intervals.

Dialysis was performed for four hours with $1.3 \mathrm{~m}^{2}$ hollow fibre kidneys. For the first session equal numbers of patients were randomly assigned by a technician who was unaware of the study design to receive dialysis either with an acetate buffered dialysis fluid (sodium $135 \mathrm{mmol} / 1$, potassium 1.4 $\mathrm{mmol} / \mathrm{l}$, calcium $1.8 \mathrm{mmol} / \mathrm{l}$, magnesium $0.5 \mathrm{mmol} / \mathrm{l}$, chloride $105 \mathrm{mmol} / \mathrm{l}$, acetate $35 \mathrm{mmol} / \mathrm{l}$, lactate $1.3 \mathrm{mmol} / \mathrm{l}$ ) or with a bicarbonate buffered dialysis fluid (sodium $138 \mathrm{mmol} / \mathrm{l}$, potassium $2 \mathrm{mmol} / \mathrm{l}$, calcium 1.75 $\mathrm{mmol} / \mathrm{l}$, magnesium $0.5 \mathrm{mmol} / \mathrm{l}$, chloride $109 \mathrm{mmol} / \mathrm{l}$, bicarbonate 32 $\mathrm{mmol} / \mathrm{l}$, acetate $3 \mathrm{mmol} / \mathrm{l}$ ). Dialysis was performed with the alternative dialysis fluid during the next two sessions and with the original dialysis fluid in the final session. The doctor but not the patient was aware of which form of dialysis was given. Fluid was removed at a continuous rate throughout dialysis by a volumetric ultrafiltration control (Dylade $\mathrm{E}$ series). Muscle cramps, vomiting, and symptomatic hypotension were treated by reducing the ultrafiltration rate and if this was unsuccessful by administering $100 \mathrm{ml}$ physiological saline.

Measurements of venous tone and blood flow in the forearm were obtained by venous occlusion plethysmography with mercury in Silastic strain gauges in the arm without the fistula. All measurements were made with the patients supine. Venous tone was measured as the increase in volume of the forearm when a venous occlusion cuff was applied around the upper arm, causing the venous bed to fill. ${ }^{4}$ We used a venous occlusion pressure of $40 \mathrm{~mm} \mathrm{Hg}$. Vascular resistance was calculated by dividing mean arterial pressure by blood flow in the forearm, which was measured by standard techniques. ${ }^{5}$ Arterial blood pressure was measured by auscultation in the same arm (diastolic pressure taken at phase V) immediately before measurement of blood flow. All measurements were made at an environmental temperature of $20-24^{\circ} \mathrm{C}$.

Patients rested supine for 15 minutes before measurement of blood flow and venous tone in the forearm, heart rate, and blood pressure. Haemodialysis was then performed, and measurements were repeated at 15 minute intervals during dialysis and 30 minutes after dialysis. Packed cell volume and plasma concentrations of sodium, potassium, bicarbonate, urea, and creatinine were measured before and at the end of each dialysis session.

Because patients had dialysis with each type of fluid twice, measurements made for each fluid were averaged. Order effects and their interaction with the type of dialysis were not analysed, but the tables present the data for each patient in the order they were obtained so that order effects can be assimilated. Patients were grouped in the tables according to whether they first received dialysis with acetate or bicarbonate fluid. They were actually studied in the chronological order case $1,5,2,6,7,3,8,4$.

To determine the significance of changes in haemodynamic variables during haemodialysis the slopes of regression lines of each variable with time during each dialysis session were calculated for each patient by the least squares method. Whether regression slopes differed significantly from zero during dialysis with either acetate or bicarbonate fluid was tested by applying the signed rank sum test to the average of the two slopes that had been calculated for each type of dialysis for each patient. Differences between the two forms of dialysis were compared for each patient with the Wilcoxon matched pairs signed ranks test.

Changes in haemodynamic variables from before to 30 minutes after each type of dialysis and biochemical variables (except creatinine concentration) from before to the end of each type of dialysis were analysed with Student's paired $t$ test. Creatinine values showed considerable variability and were therefore analysed with the Wilcoxon matched pairs signed ranks test.

\section{Results}

Table I shows changes in biochemical variables from before to the end of dialysis. Plasma concentrations of creatinine and potassium had fallen to a similar extent at the end of dialysis with acetate and bicarbonate fluids, and the plasma sodium concentration had not changed significantly at the end of either form of dialysis. The mean increase in plasma bicarbonate concentration was $3.63(\mathrm{SE} 0.92) \mathrm{mmol} / \mathrm{l}(\mathrm{p}<0.01)$ at the end of dialysis with acetate 
TABLE I-Changes in plasma sodium, potassium, bicarbonate, and creatinine concentrations and in packed cell volume and weight during dialysis

\begin{tabular}{|c|c|c|c|c|c|c|}
\hline Type of dialysis fluid & Sodium $(\mathrm{mmol} / \mathrm{l})$ & Potassium (mmol/l) & Bicarbonate $(\mathrm{mmol} / \mathrm{l})$ & Creatinine $(\mu \mathrm{mol} / \mathrm{l})$ & Packed cell volume & Weight (kg) \\
\hline $\begin{array}{l}\text { Acetate } \\
\text { Bicarbonate } \\
\text { Bicarbonate } \\
\text { Acetate }\end{array}$ & $\begin{array}{l}-4 \\
-3 \\
-1 \\
-6\end{array}$ & $\begin{array}{l}-0.9 \\
-1.5 \\
-1.1 \\
-1.7\end{array}$ & $\begin{array}{c}\text { Case I } \\
3 \\
11 \\
10 \\
5\end{array}$ & $\begin{array}{l}-636 \\
-750 \\
-505 \\
-637\end{array}$ & $\begin{array}{l}0.03 \\
0.01 \\
0.02 \\
0.02\end{array}$ & $\begin{array}{l}-2.3 \\
-1.8 \\
-1.5 \\
-1.5\end{array}$ \\
\hline Mean difference ${ }^{\star}$ & -3 & 0 & $-6 \cdot 5$ & -9 & 0.01 & $-0 \cdot 25$ \\
\hline $\begin{array}{l}\text { Acetate } \\
\text { Bicarbonate } \\
\text { Bicarbonate } \\
\text { Acetate }\end{array}$ & $\begin{array}{l}-5 \\
-6 \\
-2 \\
-6\end{array}$ & $\begin{array}{l}-1 \cdot 2 \\
-1 \\
-0.4 \\
-0.4\end{array}$ & $\begin{array}{c}\text { Case } 2 \\
3 \\
3 \\
6 \\
4\end{array}$ & $\begin{array}{l}-911 \\
-951 \\
-960 \\
-870\end{array}$ & $\begin{array}{l}0.04 \\
0.04 \\
0.04 \\
0.06\end{array}$ & $\begin{array}{l}-2 \cdot 5 \\
-2 \cdot 0 \\
-2 \cdot 2 \\
-2 \cdot 2\end{array}$ \\
\hline Mean difference ${ }^{\star}$ & $-1 \cdot 5$ & $-0 \cdot 1$ & -1 & 65 & 0.01 & $-0 \cdot 25$ \\
\hline $\begin{array}{l}\text { Acetate } \\
\text { Bicarbonate } \\
\text { Bicarbonate } \\
\text { Acetate }\end{array}$ & $\begin{array}{l}0 \\
1 \\
3 \\
1\end{array}$ & $\begin{array}{l}-0.9 \\
-0.8 \\
-0.9 \\
-1\end{array}$ & $\begin{array}{c}\text { Case } 3 \\
-1 \\
3 \\
2 \\
2\end{array}$ & $\begin{array}{l}-313 \\
-545 \\
-457 \\
-367\end{array}$ & $\begin{array}{l}0.05 \\
0.02 \\
0.02 \\
0.02\end{array}$ & $\begin{array}{l}-1 \cdot 7 \\
-2 \cdot 1 \\
-1 \cdot 7 \\
-1 \cdot 2\end{array}$ \\
\hline Mean difference* & $-1 \cdot 5$ & $-0 \cdot 1$ & -2 & 161 & 0.015 & 0.45 \\
\hline $\begin{array}{l}\text { Acetate } \\
\text { Bicarbonate } \\
\text { Bicarbonate } \\
\text { Acetate }\end{array}$ & $\begin{array}{r}0 \\
-2 \\
0 \\
-4\end{array}$ & $\begin{array}{l}-3 \cdot 7 \\
-2 \cdot 4 \\
-1.8 \\
-2.6\end{array}$ & $\begin{array}{c}\text { Case } 4 \\
2 \\
10 \\
9 \\
2\end{array}$ & $\begin{array}{r}-1023 \\
-893 \\
-1152 \\
-983\end{array}$ & $\begin{array}{l}0.02 \\
0.02 \\
0.01 \\
0.02\end{array}$ & $\begin{array}{l}-1 \cdot 4 \\
-1 \cdot 7 \\
-2 \cdot 1 \\
-1 \cdot 3\end{array}$ \\
\hline Mean difference ${ }^{\star}$ & -1 & -1.05 & $-7 \cdot 5$ & $19 \cdot 5$ & 0.005 & 0.55 \\
\hline $\begin{array}{l}\text { Bicarbonate } \\
\text { Acetate } \\
\text { Acetate } \\
\text { Bicarbonate }\end{array}$ & $\begin{array}{r}-2 \\
-2 \\
0 \\
-5\end{array}$ & $\begin{array}{l}-1 \\
-1 \cdot 1 \\
-2 \cdot 2 \\
-1\end{array}$ & $\begin{array}{c}\text { Case } 5 \\
7 \\
1 \\
5 \\
9\end{array}$ & $\begin{array}{l}-473 \\
-445 \\
-515 \\
-490\end{array}$ & $\begin{array}{l}0.02 \\
0.02 \\
0.04 \\
0 \cdot 02\end{array}$ & $\begin{array}{l}-2 \cdot 5 \\
-3 \cdot 4 \\
-2 \cdot 7 \\
-1 \cdot 7\end{array}$ \\
\hline Mean difference ${ }^{\star}$ & $2 \cdot 5$ & -0.65 & -5 & 1.5 & 0.01 & -0.95 \\
\hline $\begin{array}{l}\text { Bicarbonate } \\
\text { Acetate } \\
\text { Acetate } \\
\text { Bicarbonate }\end{array}$ & $\begin{array}{r}-2 \\
0 \\
-3 \\
-1\end{array}$ & $\begin{array}{l}-1 \cdot 1 \\
-1 \cdot 5 \\
-2 \\
-1 \cdot 6\end{array}$ & $\begin{array}{c}\text { Case } 6 \\
5 \\
4 \\
3 \\
3\end{array}$ & $\begin{array}{l}-577 \\
-524 \\
-679 \\
-823\end{array}$ & $\begin{array}{l}0.01 \\
0.01 \\
0.01 \\
0.01\end{array}$ & $\begin{array}{l}-2 \cdot 3 \\
-2 \cdot 4 \\
-2 \cdot 4 \\
-1 \cdot 8\end{array}$ \\
\hline Mean difference ${ }^{\star}$ & 0 & $-0 \cdot 4$ & -0.5 & $98 \cdot 5$ & 0 & -0.35 \\
\hline $\begin{array}{l}\text { Bicarbonate } \\
\text { Acetate } \\
\text { Acetate } \\
\text { Bicarbonate }\end{array}$ & $\begin{array}{l}2 \\
2 \\
1 \\
0\end{array}$ & $\begin{array}{l}-1.8 \\
-0.9 \\
-1.4 \\
-1.4\end{array}$ & $\begin{array}{c}\text { Case } 7 \\
9 \\
2 \\
4 \\
8\end{array}$ & $\begin{array}{l}-1134 \\
-1077 \\
-1183 \\
-1079\end{array}$ & $\begin{array}{l}0.02 \\
0.02 \\
0.02 \\
0.02\end{array}$ & $\begin{array}{l}-1 \cdot 2 \\
-2 \\
-2 \cdot 4 \\
-2 \cdot 6\end{array}$ \\
\hline Mean difference ${ }^{\star}$ & 0.5 & 0.45 & $-5 \cdot 5$ & $-23 \cdot 5$ & 0 & $-0 \cdot 3$ \\
\hline $\begin{array}{l}\text { Bicarbonate } \\
\text { Acetate } \\
\text { Acetate } \\
\text { Bicarbonate }\end{array}$ & $\begin{array}{l}0 \\
3 \\
0 \\
0\end{array}$ & $\begin{array}{l}-2 \cdot 6 \\
-2 \cdot 8 \\
-3 \cdot 1 \\
-1 \cdot 7\end{array}$ & $\begin{array}{c}\text { Case } 8 \\
9 \\
11 \\
8 \\
10\end{array}$ & $\begin{array}{l}-562 \\
-894 \\
-671 \\
-600\end{array}$ & $\begin{array}{l}0 \cdot 02 \\
0.01 \\
0.02 \\
0 \cdot 02\end{array}$ & $\begin{array}{l}-2 \cdot 2 \\
-2 \cdot 5 \\
-2 \cdot 6 \\
-2 \cdot 5\end{array}$ \\
\hline Mean difference ${ }^{\star}$ & 1.5 & $-0 \cdot 8$ & 0 & $-201 \cdot 5$ & -0.005 & $-0 \cdot 2$ \\
\hline $\begin{array}{l}\text { Mean }(\mathrm{SE}) \text { change with } \\
\text { acetate fluid }(n=8) \\
\text { Mean }(\mathrm{SE}) \text { change with } \\
\text { bicarbonate fluid }(n=8) \\
\text { Mean }(\mathrm{SE}) \text { difference }{ }^{\star} \\
(\mathrm{n}=8)\end{array}$ & $\begin{array}{l}-1.44(0.95) \\
-1.13(0.74) \\
-0.31(0.63)\end{array}$ & $\begin{array}{l}-1.71 \dagger(0.31) \\
-1.38 \ddagger(0.19) \\
-0.33(0.17)\end{array}$ & $\begin{array}{l}\text { Overall changes } \\
\qquad \begin{array}{r}3.63 \dagger(0.93) \\
7.13 \ddagger(1.06) \\
-3.5 \dagger(1.0)\end{array}\end{array}$ & $\begin{array}{r}-733 \oint(94 \cdot 8) \\
-747 \Phi(87 \cdot 0) \\
13.9(37 \cdot 8)\end{array}$ & $\begin{array}{l}0.026 \ddagger(0.005) \\
0.02 \ddagger(0.003) \\
0.006(0.002)\end{array}$ & $\begin{array}{l}-2 \cdot 16 \ddagger(0.20) \\
-1.99 \ddagger(0.07) \\
-0 \cdot 16(0 \cdot 16)\end{array}$ \\
\hline
\end{tabular}

* Mean for dialysis with acetate fluid minus mean for dialysis with bicarbonate fluid.

$t \mathrm{p}<0.01, \neq \mathrm{p}<0.001$ with Student's paired $t$ test. $\$ \mathrm{p}<0.01$ with Wilcoxon matched pair signed ranks test.

fluid and $7 \cdot 13(1.06) \mathrm{mmol} / \mathrm{l}(\mathrm{p}<0.001)$ at the end of dialysis with bicarbonate fluid. This increase was greater after dialysis with bicarbonate fluid (mean difference $3.5 \mathrm{mmol} / 1$ within patient $\mathrm{SE} 1.0 ; \mathrm{p}<0.01$ ). The mean increase in packed cell volume was $0.026(0.005)(p<0.001)$ at the end of dialysis with acetate fluid and $0.02(0.003)(\mathrm{p}<0.001)$ at the end of dialysis with bicarbonate fluid. The mean difference in the increases in packed cell volume within patients between dialysis with acetate and bicarbonate fluids was $0.006(0.002)(95 \%$ confidence interval -0.0001 to 0.011$)$. Mean weight loss was $2 \cdot 2(0 \cdot 2) \mathrm{kg}$ during dialysis with acetate fluid and $2 \cdot 0(0 \cdot 1) \mathrm{kg}$ during dialysis with bicarbonate fluid.

Table II shows variable haemodynamic changes from before dialysis to 30 minutes after dialysis. The mean increase in heart rate 30 minutes after dialysis was $6.9(2.3)$ beats/min $(\mathrm{p}<0.02)$ for dialysis with acetate fluid and $5 \cdot 3(2 \cdot 1)$ beats $/ \mathrm{min}(\mathrm{p}<0.05)$ for dialysis with bicarbonate fluid. Mean arterial blood pressure had fallen by a mean of $8.6(1.9) \mathrm{mm} \mathrm{Hg}(\mathrm{p}<0.01) 30$ minutes after dialysis with acetate fluid and $5.1(1.8) \mathrm{mm} \mathrm{Hg}(\mathrm{p}<0.05) 30$ minutes after dialysis with bicarbonate fluid. The mean difference in the fall in blood pressure within patients between dialysis with acetate and bicarbonate fluid was $2 \cdot 3(2 \cdot 3) \mathrm{mm} \mathrm{Hg}(95 \%$ confidence interval $-2 \cdot 4$ to $7 \cdot 0)$

Venodilatation was present in the forearm after dialysis with both acetate and bicarbonate fluid. The mean rise in the index of venous tone was 0.65 
$(0.11) \mathrm{ml} / \mathrm{dl}$ over $40 \mathrm{~mm} \mathrm{Hg}(\mathrm{p}<0.001)$ for dialysis with acetate fluid and $0.64(0.23) \mathrm{ml} / \mathrm{dl}$ over $40 \mathrm{~mm} \mathrm{Hg}(\mathrm{p}<0.05)$ for dialysis with bicarbonate fluid. The greater variability in the index of venous tone 30 minutes after dialysis with bicarbonate was accounted for by one patient (case 2) developing pronounced venoconstriction after one session of dialysis with bicarbonate fluid. Vascular resistance in the forearm (measured in arbitrary units) had increased by an average of 4.54 (2.52) (95\% confidence interval
-1.4 to 10.5$)$ after dialysis with acetate fluid and by $5.87(1.70)(95 \%$ confidence interval 1.85 to $9.87 ; \mathrm{p}<0.02$ ) after dialysis with bicarbonate fluid.

The data for individual patients during each of the four treatment periods were examined to confirm that the changes in variables were linear. Heart rate increased at a mean rate of $2.6(0.84)$ beats $/ \mathrm{min} / \mathrm{hour}$ during dialysis with acetate fluid $(\mathrm{p}<0.05)$ and $2.6(0.89)$ beats $/ \mathrm{min} /$ hour during dialysis

TABLE II-Haemodynamic changes from before to 30 minutes after dialysis

\begin{tabular}{|c|c|c|c|c|c|c|c|c|}
\hline \multirow[b]{2}{*}{ Type of dialysis fluid } & \multicolumn{2}{|c|}{ Heart rate (beats/min) } & \multicolumn{2}{|c|}{$\begin{array}{c}\text { Mean arterial pressure } \\
(\mathrm{mm} \mathrm{Hg})\end{array}$} & \multicolumn{2}{|c|}{$\begin{array}{l}\text { Venous tone in forearm } \\
(\mathrm{m} / \mathrm{dl} \text { over } 40 \mathrm{~mm} \mathrm{Hg})\end{array}$} & \multicolumn{2}{|c|}{$\begin{array}{l}\text { Vascular resistance in forearm } \\
\text { (arbitrary units) }\end{array}$} \\
\hline & Before dialysis & Change & Before dialysis & Change & Before dialysis & Change & Before dialysis & Change \\
\hline $\begin{array}{l}\text { Acetate } \\
\text { Bicarbonate } \\
\text { Bicarbonate } \\
\text { Acetate }\end{array}$ & $\begin{array}{l}76 \\
72 \\
68 \\
72\end{array}$ & $\begin{array}{r}4 \\
4 \\
14 \\
8\end{array}$ & $\begin{array}{ll} & C o \\
97 & \\
91 & \\
98 & \\
93 & \end{array}$ & $\begin{array}{r}s e l \\
-8 \\
8 \\
-6 \\
-25\end{array}$ & $\begin{array}{l}1.1 \\
1.6 \\
1.8 \\
2.0\end{array}$ & $\begin{array}{l}0.5 \\
0.9 \\
0.9 \\
0.8\end{array}$ & $\begin{array}{l}16 \cdot 0 \\
24 \cdot 7 \\
16 \cdot 3 \\
17 \cdot 4\end{array}$ & $\begin{array}{r}9 \cdot 3 \\
0.5 \\
14.4 \\
6.8\end{array}$ \\
\hline Mean difference ${ }^{\star}$ & 4 & -3 & 0.5 & $-15 \cdot 5$ & -0.15 & $-0 \cdot 25$ & $-3 \cdot 8$ & 0.6 \\
\hline $\begin{array}{l}\text { Acetate } \\
\text { Bicarbonate } \\
\text { Bicarbonate } \\
\text { Acetate }\end{array}$ & $\begin{array}{l}72 \\
64 \\
60 \\
60\end{array}$ & $\begin{array}{r}12 \\
0 \\
32 \\
28\end{array}$ & $\begin{array}{rr}117 & C o \\
113 & \\
112 & \\
95 & \end{array}$ & $\begin{array}{r}\text { se } 2 \\
-14 \\
-10 \\
10 \\
0\end{array}$ & $\begin{array}{l}2 \cdot 7 \\
2 \cdot 8 \\
3 \cdot 2 \\
2 \cdot 9\end{array}$ & $\begin{array}{r}0.1 \\
0.2 \\
-1.5 \\
0.5\end{array}$ & $\begin{array}{l}28 \cdot 5 \\
22 \cdot 5 \\
20 \cdot 4 \\
17 \cdot 2\end{array}$ & $\begin{array}{r}-1.5 \\
3.2 \\
6.7 \\
9.8\end{array}$ \\
\hline Mean difference & 4 & 4 & $-6 \cdot 5$ & -7 & -0.2 & 0.95 & 1.4 & -0.8 \\
\hline $\begin{array}{l}\text { Acetate } \\
\text { Bicarbonate } \\
\text { Bicarbonate } \\
\text { Acetate }\end{array}$ & $\begin{array}{l}78 \\
76 \\
76 \\
80\end{array}$ & $\begin{array}{l}2 \\
4 \\
8 \\
8\end{array}$ & $\begin{array}{l}86 \\
85 \\
95 \\
87\end{array}$ & $\begin{array}{r}\text { se } 3 \\
-5 \\
5 \\
-16 \\
-12\end{array}$ & $\begin{array}{l}1.7 \\
1.9 \\
1.5 \\
2.3\end{array}$ & $\begin{array}{l}0.3 \\
0.7 \\
1.9 \\
1.2\end{array}$ & $\begin{array}{l}37 \cdot 4 \\
36 \cdot 8 \\
39 \cdot 7 \\
29 \cdot 9\end{array}$ & $\begin{array}{r}16 \cdot 4 \\
4 \cdot 1 \\
1 \cdot 7 \\
0 \cdot 2\end{array}$ \\
\hline Mean difference ${ }^{\star}$ & 3 & -1 & $-3 \cdot 5$ & -3 & 0.3 & -0.55 & $-4 \cdot 6$ & $5 \cdot 4$ \\
\hline $\begin{array}{l}\text { Acetate } \\
\text { Bicarbonate } \\
\text { Bicarbonate } \\
\text { Acetate }\end{array}$ & $\begin{array}{l}76 \\
62 \\
60 \\
68\end{array}$ & $\begin{array}{r}8 \\
-2 \\
8 \\
0\end{array}$ & $\begin{array}{r}95 \\
103 \\
109 \\
100\end{array}$ & $\begin{array}{r}\text { se } 4 \\
0 \\
-1 \\
-27 \\
-13\end{array}$ & $\begin{array}{l}1.5 \\
2.0 \\
3.0 \\
2.8\end{array}$ & $\begin{array}{r}0.8 \\
-0.3 \\
0.8 \\
0.1\end{array}$ & $\begin{array}{l}23 \cdot 3 \\
22 \cdot 2 \\
26 \cdot 5 \\
25 \cdot 0\end{array}$ & $\begin{array}{r}1 \cdot 0 \\
0 \cdot 6 \\
9 \cdot 9 \\
-0 \cdot 2\end{array}$ \\
\hline Mean difference & 11 & 1 & $-8 \cdot 5$ & 7.5 & -0.35 & 0.2 & -0.2 & $-4 \cdot 85$ \\
\hline $\begin{array}{l}\text { Bicarbonate } \\
\text { Acetate } \\
\text { Acetate } \\
\text { Bicarbonate }\end{array}$ & $\begin{array}{l}68 \\
60 \\
64 \\
68\end{array}$ & $\begin{array}{r}10 \\
12 \\
4 \\
8\end{array}$ & $\begin{array}{rr} & C o \\
107 & \\
101 & \\
97 & \\
113 & \end{array}$ & $\begin{array}{r}\text { se } 5 \\
-16 \\
-2 \\
-1 \\
-2\end{array}$ & $\begin{array}{l}2 \cdot 1 \\
2 \cdot 1 \\
2 \cdot 0 \\
1 \cdot 8\end{array}$ & $\begin{array}{l}1.5 \\
0.2 \\
0.5 \\
0.5\end{array}$ & $\begin{array}{l}19 \cdot 9 \\
30 \cdot 0 \\
24 \cdot 2 \\
19 \cdot 5\end{array}$ & $\begin{array}{c}0 \\
25 \cdot 2 \\
12 \cdot 7 \\
14 \cdot 2\end{array}$ \\
\hline Mean difference* & -6 & -1 & -11 & $7 \cdot 5$ & 0.1 & -0.65 & $7 \cdot 4$ & 11.85 \\
\hline $\begin{array}{l}\text { Bicarbonate } \\
\text { Acetate } \\
\text { Acetate } \\
\text { Bicarbonate }\end{array}$ & $\begin{array}{l}88 \\
80 \\
88 \\
84\end{array}$ & $\begin{array}{r}0 \\
2 \\
-8 \\
0\end{array}$ & $\begin{array}{ll} & C o \\
118 & \\
115 & \\
123 & \\
123 & \end{array}$ & $\begin{array}{r}3 \\
-6 \\
-1 \\
-4\end{array}$ & $\begin{array}{l}1.0 \\
1.1 \\
1.4 \\
1.2\end{array}$ & $\begin{array}{l}0 \cdot 2 \\
1 \cdot 0 \\
0 \cdot 3 \\
0 \cdot 3\end{array}$ & $\begin{array}{l}29 \cdot 5 \\
38 \cdot 4 \\
31.5 \\
24.5\end{array}$ & $\begin{array}{r}21 \cdot 1 \\
-8 \cdot 9 \\
2 \cdot 3 \\
11 \cdot 5\end{array}$ \\
\hline Mean difference & -2 & -3 & -1.5 & -3 & $0 \cdot 15$ & $0 \cdot 4$ & 8.0 & $-19 \cdot 6$ \\
\hline $\begin{array}{l}\text { Bicarbonate } \\
\text { Acetate } \\
\text { Acetate } \\
\text { Bicarbonate }\end{array}$ & $\begin{array}{l}60 \\
58 \\
64 \\
62\end{array}$ & $\begin{array}{r}0 \\
2 \\
16 \\
-2\end{array}$ & $\begin{array}{ll}109 & C o \\
121 & \\
115 & \\
119 & \end{array}$ & $\begin{array}{r}\text { se } 7 \\
-10 \\
-9 \\
-8 \\
-1\end{array}$ & $\begin{array}{l}1 \cdot 6 \\
2 \cdot 2 \\
2 \cdot 9 \\
2 \cdot 3\end{array}$ & $\begin{array}{l}0.5 \\
1.6 \\
0 \\
2.0\end{array}$ & $\begin{array}{l}25 \cdot 3 \\
31 \cdot 8 \\
23 \cdot 1 \\
20 \cdot 8\end{array}$ & $\begin{array}{r}0 \\
5.5 \\
-5 \cdot 4 \\
2.8\end{array}$ \\
\hline Mean difference & 0 & 10 & 4 & -3 & 0.6 & $-0 \cdot 45$ & $4 \cdot 4$ & $-1 \cdot 35$ \\
\hline $\begin{array}{l}\text { Bicarbonate } \\
\text { Acetate } \\
\text { Acetate } \\
\text { Bicarbonate }\end{array}$ & $\begin{array}{l}90 \\
88 \\
90 \\
90\end{array}$ & $\begin{array}{l}0 \\
8 \\
4 \\
0\end{array}$ & $\begin{array}{ll} & C c \\
81 & \\
97 & \\
89 & \\
89 & \end{array}$ & $\begin{array}{r}\text { se } 8 \\
2 \\
-17 \\
-16 \\
-16\end{array}$ & $\begin{array}{l}1 \cdot 3 \\
1 \cdot 4 \\
1 \cdot 3 \\
1 \cdot 5\end{array}$ & $\begin{array}{l}1 \cdot 3 \\
1 \cdot 4 \\
1 \cdot 1 \\
0.3\end{array}$ & $\begin{array}{l}20 \cdot 3 \\
21 \cdot 6 \\
20 \cdot 2 \\
24 \cdot 8\end{array}$ & $\begin{array}{r}6 \cdot 4 \\
-4 \cdot 6 \\
4 \cdot 0 \\
-3 \cdot 2\end{array}$ \\
\hline Mean difference & -1 & 6 & 8 & -9.5 & -0.05 & 0.45 & $-1 \cdot 7$ & $-1 \cdot 9$ \\
\hline $\begin{array}{l}\text { Mean (SE) of dialysis with } \\
\text { acetate }(n=8) \\
\text { Mean ( }(E) \text { of dialysis with } \\
\text { bicarbonate }(n=8) \\
\text { Mean }(S E) \text { of difference } \\
(n=8)\end{array}$ & $\begin{array}{r}73.4(3.6) \\
71 \cdot 8(4.0) \\
1.6(1.8)\end{array}$ & $\begin{array}{l}6.9 \ddagger(2 \cdot 3) \\
5.3 †(2 \cdot 1) \\
1.6(1.6)\end{array}$ & $\begin{array}{r}\text { Overal } \\
101 \cdot 8(4 \cdot 1) \\
104 \cdot 1(4 \cdot 5) \\
-2 \cdot 3(2 \cdot 3)\end{array}$ & $\begin{array}{l}\text { changes } \\
-8.6 \oint(1.9) \\
-5.1 \dagger(1.8) \\
-3.3(2 \cdot 8)\end{array}$ & $\begin{array}{l}1.96(0.20) \\
1.91(0.21) \\
0.5(0.10)\end{array}$ & $\begin{array}{l}0.65 \|(0.11) \\
0.64 \dagger(0.23) \\
0.01(0.20)\end{array}$ & $\begin{array}{r}25 \cdot 98(2 \cdot 19) \\
24 \cdot 61(2 \cdot 11) \\
1 \cdot 36(1 \cdot 71)\end{array}$ & $\begin{array}{r}4.54(2.52) \\
5.87 \ddagger(1.70) \\
-1.33(3.19)\end{array}$ \\
\hline
\end{tabular}

* Mean for dialysis with acetate fluid minus mean for dialysis with bicarbonate fluid.

$+\mathrm{p}<0.05, \neq \mathrm{p}<0.02, \S \mathrm{p}<0.01, \| \mathrm{p}<0.001$ with Student's paired $t$ test. 
with bicarbonate fluid $(p<0.05)$. Mean arterial blood pressure fell at a mean rate of $3.9(0.90) \mathrm{mm} \mathrm{Hg} / \mathrm{hour}$ during dialysis with acetate fluid $(\mathrm{p}<0.01)$ and $1.4(0.52) \mathrm{mm} \mathrm{Hg} /$ hour during dialysis with bicarbonate fluid $(\mathrm{p}<0.05)$. The rate of fall was significantly greater during dialysis with acetate than with bicarbonate fluid $(\mathrm{p}<0.02)$. The index of venous tone rose at a mean rate of $0.23(0.05) \mathrm{ml} / \mathrm{dl}$ over $40 \mathrm{~mm} \mathrm{Hg} /$ hour during dialysis with acetate fluid $(\mathrm{p}<0.01)$ and $0.20(0.05) \mathrm{ml} / \mathrm{dl}$ over $40 \mathrm{~mm} \mathrm{Hg} / \mathrm{hour}$ during dialysis with bicarbonate fluid $(p<0.01)$. Vascular resistance in the forearm increased at a mean rate of $3.6(1 \cdot 12)$ units/hour during dialysis with acetate fluid $(\mathrm{p}<0.02)$ and $4.5(1.48)$ units/hour during dialysis with bicarbonate fluid $(\mathrm{p}<0.01)$. There were no significant differences in the changes in venous tone or vascular resistance between dialysis with acetate and bicarbonate fluid.

\section{Discussion}

The results of this study point to important abnormalities of the control of the peripheral circulation during dialysis that may contribute to the development of hypotension. The normal responses to a reduction in plasma volume such as that occurring during dialysis are venoconstriction and an increase in peripheral vascular resistance. ${ }^{7}$ Both of these mechanisms help to prevent a fall in blood pressure. The venoconstriction compensates for the reduced central plasma volume and maintains cardiac filling pressures and thereby cardiac output. The increase in peripheral vascular resistance helps maintain blood pressure despite a fall in cardiac output. Our patients showed a normal increase in peripheral vascular resistance during dialysis, but paradoxically their veins dilated. This venodilatation would accentuate the fall in central blood volume and lead to a fall in cardiac filling pressures and cardiac output and then to hypotension. Evidence supporting this as the mechanism of hypotension during dialysis is that an infusion of fluid often corrects the hypotension.

The reason for the abnormal dilatation of the venous bed is unclear. It occurred equally during both types of dialysis and therefore is not due to the vasodilating properties of acetate. It does not seem to be due to autonomic dysfunction as there were appropriate changes in heart rate and peripheral resistance during dialysis, and the veins actively dilated. It is more likely that the correction of the metabolic derangements of renal failure withdrew a stimulus to venoconstriction. Whatever the mechanism, simple measures-for example, wearing support stockings-aimed at reducing pooling of blood in peripheral veins may prevent hypotension induced by dialysis.

This study was supported by a grant from the Addenbrooke's Kidney Patients' Association.

\section{References}

1 Henrich WL. Hemodynamic instability during hemodialysis. Kidney Int 1986;30:605-12. 2 Mansell MA, Wing AJ. Acetate or bicarbonate for haemodialysis. Br Med $\mathcal{J}$ 1983;287:308-9. 3 Kinet J, Soyeur D, Balland N, Saint-Remy M, Collignon P, Godon J. Hemodynamic study of hypotension during hemodialysis. Kidney Int 1982;21:868-76.

4 Bevegard BS, Shepherd JT. Changes in the tone of limb veins during supine exercise. J Appl Physiol 1965;20:1-8.

5 Greenfield ADM, Whitney RI, Mowbray FR. Methods for the investigation of peripheral blood flow. Br Med Bull 1963;19:101-4.

6 Hsu CH, Swartz RD, Somermeyer MG, Raj A. Bicarbonate hemodialysis: influence on plasma refilling and hemodynamic stability. Nephron 1984;38:202-8.

7 Abboud FM, Heistad DD, Allyn LM, Schmid PG. Reflex control of the peripheral circulation. Prog Cardiovasc Dis 1976;18:371-403.

\title{
Management of asthma in hospital: a prospective audit
}

\author{
C E BUCKNALL，C ROBERTSON，F MORAN， R D STEVENSON
}

\begin{abstract}
In a prospective study of management of asthma in hospital patients with acute asthma admitted to a single hospital over a calendar year were surveyed. Altogether 157 out of 194 admissions $(81 \%)$ were studied. The patients (16 of whom had been admitted twice and one three times) were interviewed at home about two weeks after discharge, and their hospital records were reviewed. When interviewed an appreciable proportion of patients said that their asthma had been poorly controlled after their discharge from the hospital: 54 reported regular sleep disturbance due to wheeze, 78 tightness of the chest in the morning, and 77 wheeze after climbing one flight of stairs. Patients had been described on admission as having had
\end{abstract}

Department of Respiratory Medicine, Glasgow Royal Infirmary, Glasgow G4 OSF

C E BUCKNALL, MB, MRCP, registrar

F MORAN, MB, FRCP, consultant physician

R D STEVENSON, MD, FRCP, consultant physician

Department of Mathematics, Strathclyde University, Glasgow G1 1XQ C ROBERTSON, PHD, lecturer

Correspondence to: Dr C E Bucknall, Govan Health Centre, Glasgow G51 4BJ. symptoms of deteriorating asthma for a median of three days. Closer questioning of 71 patients, however, elicited that 50 had had regular symptoms indicating poor control for weeks or months. Most patients did not know how their drugs worked, and many did not have an appropriate plan of action in the event of a further attack. In all the cases studied 114 patients were treated with oral corticosteroids, only 70 had had their previous maintenance treatment increased at the time of discharge, and 107 had a follow up appointment booked for an average of three and a half weeks after discharge.

These findings show that undersupervision and undertreatment of patients with asthma are common and not confined to those dying of the condition.

\section{Introduction}

Mortality from asthma is either static ${ }^{1}$ or rising in Britain, ${ }^{2}$ with about 2000 deaths a year. Oral and inhaled corticosteroids and $\beta_{2}$ agonists, which are effective in treating asthma, have not had any impact on this figure. Several surveys of deaths from asthma have found that some factors are preventable. ${ }^{3.5}$ For example, in the British Thoracic Association's survey of deaths from asthma in two regions of England such factors existed in 77 of 90 cases: corticosteroids and bronchodilators were underused, patients were poorly supervised, and both patients and doctors underestimated 